\title{
Application of a three-dimensional fiber lay-down model to non-woven production processes
}

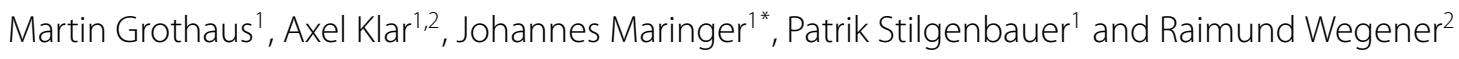

\section{"Correspondence:}

johannes.maringer@itwm.fraunhofer.de

${ }^{1}$ Technische Universität

Kaiserslautern, Gottlieb-Daimler Str.,

Kaiserslautern, 67663, Germany

Full list of author information is

available at the end of the article

\begin{abstract}
In this work we present the industrial application of fiber lay-down models that enable an efficient simulation of non-woven structures. The models describe the deposition of fibers on a moving conveyor belt with the help of stochastic differential equations on manifolds. The model parameters have to be estimated from more complex models in combination with measurements of the resulting non-woven. In the application we discuss especially a three-dimensional fiber model for a typical industrial problem from non-woven production processes.
\end{abstract}

Keywords: fiber lay-down; stochastic differential equations on manifolds; parameter identification

\section{Introduction}

Technical textiles have attracted great attention to diverse branches of industry over the last decades due to their comparatively cheap manufacturing. By overlapping thousands of individual slender fibers, random fiber webs emerge yielding non-woven materials that find applications e.g. in textile, building and hygiene industry as integral components of baby diapers, closing textiles, filters and medical devices, to name but a few. A typical method of production is given by melt-spinning processes, see Figure 1 for a corresponding plant. There melted polymers are extruded through narrow nozzles leading to plenty single fibers. Then these fibers are stretched and spun until they solidify due to cooling air streams. The latter also account for swirling of the fibers before they are laid down on a moving conveyor belt. The resulting fiber web eventually passes through several processing steps of reworking and reinforcement before the finished product is obtained. The quality of the non-woven can be measured, for example, in terms of homogeneity, basis weight, or permeability and is already determined to a great extend by the fiber deposition. Fluctuations in these properties due to stochastic influences during the production process cause difficulties for the manufacturer, such as unintended holes in filter materials. An objective in industry is the simulation of the deposited fiber web and its optimization with respect to the desired characteristics. Since in this process, raw material costs play a more decisive role as compared to the costs of procedure, the optimization aims at raw material saving that corresponds to a small value of basis weight of the non-woven, while quality assurance. The primary objective is to generate the microstructure of the technical textile given the relevant production parameters. Then, on the one hand, the

\section{黛 Springer}

○2014 Grothaus et al.; licensee Springer. This is an Open Access article distributed under the terms of the Creative Commons Attribution License (http://creativecommons.org/licenses/by/2.0), which permits unrestricted use, distribution, and reproduction in any medium, provided the original work is properly cited. 


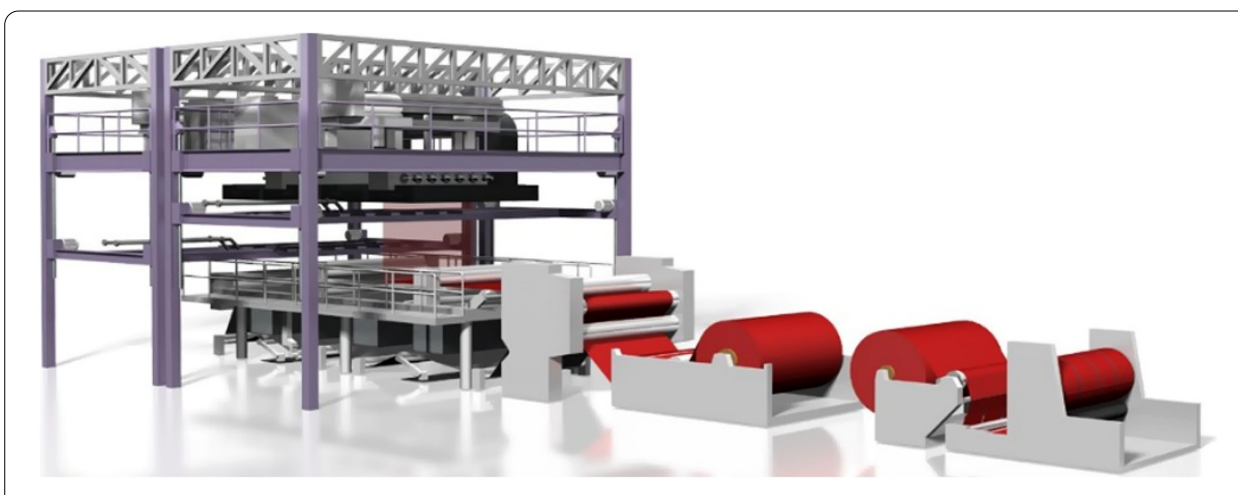

Figure 1 Plant for melt-spinning process (Photo by Oerlikon Neumag).

microstructure will be further investigated with respect to the above quality parameters. On the other hand, a microstructure simulation can be used to predict material properties of the textile. The material properties can be optimized solving the associated inverse and optimization problems. Finally, this leads to a complete virtual material design of technical textiles.

Since the mathematical treatment of the whole process at a stroke is not possible due to its complexity, a hierarchy of models that adequately describe partial aspects of the process chain has been developed in research during the last years. In [1] the conjunction of those models has been elaborated using asymptotic analysis, similarity estimates and parameter identification. More precisely, the description of the fiber dynamics in turbulent air flows has been realized by a stochastic generalized string model that has been deduced from a special Cosserat rod under Kirchhoff constraint, consult $[2,3]$ for its derivation. This provides the basis for the software tool FIDYST ${ }^{\text {a }}$ that enables the full simulation of fiber motion under the influence of surrounding turbulent air flows up to the fiber lay-down on the transport belt. In this manner, the microstructure of virtual fiber webs can be in principle generated. However, this approach is computationally expensive and the complexity is strongly affected by the number of fibers. For this reason, a class of stochastic surrogate models has been proposed in order to compute only the image of the deposited fiber web, instead of describing the full fiber dynamics that lead to this web. These surrogate models have in common the structure of a system of ordinary stochastic differential equations that enables the fast computation of a considerable number of fibers. They contain parameters that are related to the physical production process, as for example turbulence influence and fiber coiling, and that have to be identified from the full simulation of a few fibers with help of the complex string model. Based on the original two-dimensional version in [4], the surrogate models have been frequently improved within the last years. This includes the addition of a moving conveyor belt [5] or, more general, the consideration of more specific types of production processes as for instance rotational spinning processes [1]. Further enhancements are given by a smooth version in [6] where the curvatures of the fibers are taken into account, and by modelling the lay-down in three dimensions $[7,8]$. The latter has been intended as first step into a realistic generation of three-dimensional microstructures that can be used for study of flow resistance and elasticity of the nonwoven. The mathematical analysis of these models is sophisticated due to degeneracies of the associated Fokker-Planck equations. The trend to equilibrium for the original twodimensional model has been investigated using Dirichlet forms and operator semi-group 
techniques in [9], with a hypocoercivity method in [10] and with probabilistic methods in [11-13]. The higher-dimensional models have been accordingly analyzed in [13, 14]. With the help of various techniques from asymptotic analysis connections between these models have been shown and model reduction in the situation of large or small turbulence have been performed in [4-8]. A possible strategy for estimating the parameters of the two-dimensional models has been provided as a heuristic approach in [1] which has been successfully tested by FIDYST simulations of transversal as well as rotational spinning processes in the stated source. In [7] an extension of this identification strategy to the three-dimensional case has been proposed on condition that information about the fiber orientation is available. In this work we realize this approach in a real industrial problem for the first time. At the example of a pilot plant of the company Oerlikon Neumag, we discuss the calibration of the smooth three-dimensional surrogate model and illustrate the computation of corresponding fiber webs. For this we need both the full simulation of a few representative fibers with regard to the problem setting and image processing data from CT-scans of the resulting non-woven.

This paper is structured as follows. At first, we recapitulate the class of surrogate fiber lay-down models developed in the above mentioned papers. This is done in Section 2 where we use a description that can be embedded in the context of geometric Langevin equations on regular submanifolds as investigated in [15]. Via this approach, the highly geometric nature of the class of fiber lay-down models is illustrated.

In Section 3 we describe a strategy for estimating the parameters of the surrogate models. The application of the models to the production process in the pilot plant is done in Section 4 where we are able to simulate virtual fiber webs that are numerically investigated with regard to their quality characteristics.

\section{The fiber lay-down models}

As mentioned in the Introduction, in this section we recapitulate the class of surrogate models developed in $[1,7,8,13,15]$. In the surrogate fiber lay-down models the paths of the deposited fibers (as images of arc-length parametrized curves) are described by simplified stochastic differential equations. For this purpose, distinctive process parameters that influence the form of the fiber web are incorporated. These are the typical throwing ranges of the fibers and their coiling behavior, whereas the deposition itself is perturbed by some random force which is affected by the entanglement of the fibers above the laydown region. Other characteristics involve the fiber stiffness and the fiber orientation in space. Additionally, depending on the production process, different specifications can be handled using appropriate reference curves. In this work we restrict ourselves on the situation of a transversal spinning process, that means the fiber lay-down starting from fixed spinning positions onto a straight moving conveyor belt. Therein the belt velocity as well as the spinning speed are predetermined parameters given by the production process. The other parameters have to be appropriately estimated, see Section 3.

Conceptually, we distinguish between two major types of fiber lay-down models, a basic and a smooth version. For better clarity we hide the real environment of the production process for the moment and focus on the situation of a non-moving conveyor belt. The motion of the belt as well as anisotropy effects due to physical constraints can be easily included later on, when we consider the practically relevant 2D and 3D models once more 
Figure 2 Scheme $(d=2)$ of fiber curve $\xi$ with normalized tangent $\omega$.

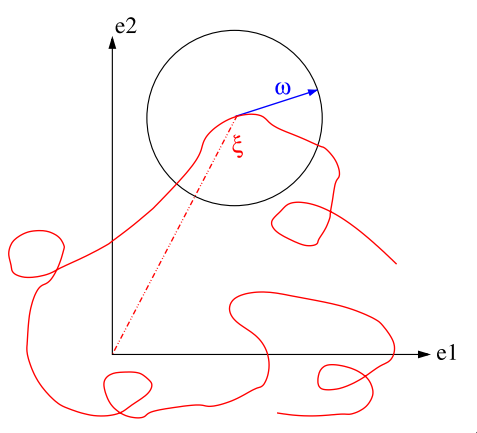

separately. In general, the basic model is formulated as a Stratonovich stochastic differential equation with state space $\mathbb{R}^{d} \times \mathbb{S}^{d-1}, d \in \mathbb{N}, d \geq 2$, of the form

$$
\begin{aligned}
\mathrm{d} \boldsymbol{\xi}_{t} & =\boldsymbol{\omega}_{t} \mathrm{~d} t, \\
\mathrm{~d} \boldsymbol{\omega}_{t} & =-\Pi\left[\boldsymbol{\omega}_{t}\right] \nabla V\left(\boldsymbol{\xi}_{t}\right) \mathrm{d} t+A \Pi\left[\boldsymbol{\omega}_{t}\right] \circ \mathrm{d} \mathbf{W}_{t},
\end{aligned}
$$

see $[8,13]$. W denotes a $d$-dimensional standard Brownian motion. For more details on manifold-valued stochastic differential equations, see e.g. [16]. Here the arc-length parametrized curve $\xi: \mathbb{R}_{0}^{+} \rightarrow \mathbb{R}^{d}$ represents one deposited fiber. $\xi$ is really arc-length parametrized since $\omega$ lives on $\mathbb{S}^{d-1}$, compare Figure 2 . The drift term in the second equation models the coiling behavior of the fiber, where $\Pi[\omega]:=I-\omega \otimes \omega$ denotes the projection of the tangent onto the sphere $\mathbb{S}^{d-1}$ with $I$ the identity matrix and $x \otimes y=x y^{T}$. The throwing ranges of the fiber can be controlled with the help of a suitable potential $V$. The second term in the equation for $\omega$ describes a Brownian motion on $\mathbb{S}^{d-1}$ with constant noise amplitude $A \in \mathbb{R}_{0}^{+}$and expresses the stochastic forces, i.e. the effect of the turbulent air flows that perturb the deposition of the fibers as desired. We remark that the basic model (1) can also be viewed as a geometric Langevin equation having spherical velocities, see [15].

Taking the curvature of the fibers into consideration, which is unavoidable for characterizing bending effects, the smooth fiber lay-down model is developed: The nondifferentiable Brownian motion in (1) is replaced by a spherical Ornstein-Uhlenbeck process, see [7] and [15]. The state space of the new model is then $\mathbb{R}^{d} \times T \mathbb{S}^{d-1}, d \in \mathbb{N}, d \geq 2$ where $T \mathbb{S}^{d-1}$ indicates the tangent bundle of the sphere, see [15]. This gives the following Stratonovich stochastic differential equation for the smooth model

$$
\begin{aligned}
& \mathrm{d} \boldsymbol{\xi}_{t}=\omega_{t} \mathrm{~d} t, \\
& \mathrm{~d} \boldsymbol{\omega}_{t}=-\Pi\left[\omega_{t}\right] \nabla V\left(\xi_{t}\right) \mathrm{d} t+\boldsymbol{v}_{t} \mathrm{~d} t, \\
& \mathrm{~d} \boldsymbol{v}_{t}=\left(\omega_{t} \otimes \boldsymbol{v}_{t}\right) \nabla V\left(\xi_{t}\right) \mathrm{d} t-\lambda \boldsymbol{v}_{t} \mathrm{~d} t-\left|\boldsymbol{v}_{t}\right|^{2} \boldsymbol{\omega}_{t} \mathrm{~d} t+\mu \Pi\left[\boldsymbol{\omega}_{t}\right] \circ \mathrm{d} \mathbf{W}_{t} .
\end{aligned}
$$

Therein, the parameter $\mu \in \mathbb{R}_{0}^{+}$takes on the role of the noise amplitude, whereas the stiffness of the fibers can be related to $\lambda \in \mathbb{R}_{0}^{+}$. The connection to (1) is given by the white noise limit (compare [7]), i.e. the basic model can be viewed as a model for non-stiff fibers.

For the efficient simulation of fiber lay-down, however, these coordinate-free formulations (1) and (2) are rather inadequate. Therefore, we consider their description in local 
coordinates. We introduce a parametrization of $\mathbb{S}^{d-1}$ or $T \mathbb{S}^{d-1}$ by

$$
\begin{aligned}
& \boldsymbol{\omega}=\boldsymbol{\tau}(\boldsymbol{\theta}):=\boldsymbol{\tau}_{d-1}\left(\theta_{1}, \ldots, \theta_{d-1}\right), \\
& \boldsymbol{v}(\boldsymbol{\theta}, \boldsymbol{\kappa})=\sum_{i=1}^{d-1} \kappa_{i} \mathbf{n}_{i}(\boldsymbol{\theta}) .
\end{aligned}
$$

Here $\boldsymbol{\tau}_{i}$ is inductively defined as

$$
\begin{aligned}
& \boldsymbol{\tau}_{i}\left(\theta_{1}, \ldots, \theta_{i}\right):=\left(\begin{array}{c}
\boldsymbol{\tau}_{i-1}\left(\theta_{1}, \ldots, \theta_{i-1}\right) \sin \theta_{i} \\
\cos \theta_{i}
\end{array}\right), \quad \theta_{i} \in(0, \pi), i \geq 2, \\
& \boldsymbol{\tau}_{1}\left(\theta_{1}\right):=\left(\begin{array}{c}
\cos \theta_{1} \\
\sin \theta_{1}
\end{array}\right), \quad \theta_{1} \in \mathbb{R} / 2 \pi \mathbb{Z},
\end{aligned}
$$

and the spherical unit vectors $\mathbf{n}_{i}$ are given by $\mathbf{n}_{i}:=\frac{1}{\left|\partial_{\theta_{i}} \boldsymbol{\tau}\right|} \partial_{\theta_{i}} \boldsymbol{\tau}$. So note that the parameters take values in $\boldsymbol{\theta} \in \mathbb{R} / 2 \pi \mathbb{Z} \times(0, \pi)^{d-2}$ (or in $\mathbb{R} / 2 \pi \mathbb{Z}$ in case $d=2$ ) and $\boldsymbol{\kappa} \in \mathbb{R}^{d-1}$ respectively.

Then the basic model in spherical polar coordinates (see [13]) reads

$$
\begin{aligned}
& \mathrm{d} \boldsymbol{\xi}_{t}=\boldsymbol{\tau}\left(\boldsymbol{\theta}_{t}\right) \mathrm{d} t, \\
& \mathrm{~d} \theta_{j, t}=\left(-\mathcal{G}_{j}\left(\boldsymbol{\theta}_{t}\right) \mathbf{n}_{j}\left(\boldsymbol{\theta}_{t}\right) \cdot \nabla V\left(\boldsymbol{\xi}_{t}\right)+\frac{A^{2}}{2} \mathcal{G}_{j}^{2}\left(\boldsymbol{\theta}_{t}\right)(j-1) \cot \theta_{j, t}\right) \mathrm{d} t+A \mathcal{G}_{j}\left(\boldsymbol{\theta}_{t}\right) \mathrm{d} W_{t}^{(j)}
\end{aligned}
$$

with $j=1, \ldots, d-1$ and $\mathcal{G}_{j}:=\left|\partial_{\theta_{j}} \tau\right|^{-1}=\prod_{i=j+1}^{d-1} \frac{1}{\sin \left(\theta_{i}\right)}$ (where the empty product in case $j=d-$ 1 is defined to be equal to 1 ). The respective smooth model in local coordinates, compare with [15], is now given by

$$
\begin{aligned}
\mathrm{d} \boldsymbol{\xi}_{t} & =\boldsymbol{\tau}\left(\boldsymbol{\theta}_{t}\right) \mathrm{d} t, \\
\mathrm{~d} \theta_{j, t} & =-\mathcal{G}_{j}\left(\boldsymbol{\theta}_{t}\right) \mathbf{n}_{j}\left(\boldsymbol{\theta}_{t}\right) \cdot \nabla V\left(\boldsymbol{\xi}_{t}\right) \mathrm{d} t+\mathcal{G}_{j}\left(\boldsymbol{\theta}_{t}\right) \kappa_{j, t} \mathrm{~d} t, \\
\mathrm{~d} \kappa_{j, t} & =\sum_{i, n=1}^{d-1} \Delta_{i n j}\left(\boldsymbol{\theta}_{t}\right) \kappa_{n, t}\left(\mathbf{n}_{i}\left(\boldsymbol{\theta}_{t}\right) \cdot \nabla V\left(\boldsymbol{\xi}_{t}\right)-\kappa_{i, t}\right) \mathrm{d} t-\lambda \kappa_{j, t} \mathrm{~d} t+\mu \mathrm{d} W_{t}^{(j)},
\end{aligned}
$$

where $j=1, \ldots, d-1$ and $\Delta_{i n j}=\mathcal{G}_{i} \partial_{\theta_{i}} \mathbf{n}_{n} \cdot \mathbf{n}_{j}$. In all these local coordinate representations $\mathbf{W}=\left(W^{(1)}, \ldots, W^{(d-1)}\right)$ denotes a standard $(d-1)$-dimensional Brownian motion. It is worth mentioning that stationary states can explicitly be computed from the respective associated Fokker-Planck equations. The equilibrium for the smooth case reads

$$
p_{s}(\boldsymbol{\xi}, \boldsymbol{\theta}, \boldsymbol{\kappa})=C e^{-(d-1) V(\xi)} \prod_{i=2}^{d-1}\left(\sin \theta_{i}\right)^{i-1} \exp \left(-\frac{\lambda}{\mu^{2}} \sum_{i=1}^{d-1} \kappa_{i}^{2}\right)
$$

with some finite constant $C>0$. Here the empty product is again equal to 1 in case $d=2$. In the basic case we obtain the stationary distribution by integrating (5) over $\boldsymbol{\kappa}$.

Now that we have the abstract framework available, we recur to the application point of view and look at the essential cases $d=2$ and $d=3$ more closely. 


\subsection{The 2D model}

Setting $d=2$ and $\theta_{1}=\alpha$ in (3) leads to the original version of the fiber lay-down models as it has been introduced in [4] as

$$
\begin{aligned}
\mathrm{d} \boldsymbol{\xi}_{t} & =\boldsymbol{\tau}\left(\alpha_{t}\right) \mathrm{d} t, \\
\mathrm{~d} \alpha_{t} & =-\mathbf{n}_{1}\left(\alpha_{t}\right) \cdot \nabla V\left(\boldsymbol{\xi}_{t}\right) \mathrm{d} t+A \mathrm{~d} W_{t} .
\end{aligned}
$$

A virtual fiber web can now be simulated by simultaneous use of this model for a large number of fibers, neglecting the influence of fiber-fiber-contact. The reference points indicating different spinning nozzles are included by adding an appropriate constant to the $\xi_{t}$-process. Using this $2 \mathrm{D}$ model, for example, the basis weight distribution of the nonwoven can straightforwardly be determined assuming a uniform thickness of the fibers.

To obtain more realistic and smoother fiber paths the Brownian motion is replaced by an Ornstein-Uhlenbeck process. Setting $d=2, \theta_{1}=\alpha$ and $\kappa_{1}=\kappa$ in (4) the smooth model reads

$$
\begin{aligned}
\mathrm{d} \boldsymbol{\xi}_{t} & =\boldsymbol{\tau}\left(\alpha_{t}\right) \mathrm{d} t, \\
\mathrm{~d} \alpha_{t} & =-\mathbf{n}_{1}\left(\alpha_{t}\right) \cdot \nabla V\left(\boldsymbol{\xi}_{t}\right) \mathrm{d} t+\kappa_{t} \mathrm{~d} t, \\
\mathrm{~d} \kappa_{t} & =-\lambda \kappa_{t} \mathrm{~d} t+\mu \mathrm{d} W_{t} .
\end{aligned}
$$

This is a natural smoother version of (6). See also [6] for a similar model.

The moving conveyor belt can be included in (6) or (7) as an additional reference curve, i.e.

$$
\mathrm{d} \xi_{t}=\boldsymbol{\tau}\left(\alpha_{t}\right) \mathrm{d} t+\nu \mathbf{e}_{1} \mathrm{~d} t
$$

gives the deviation of the fiber from a reference point determined by the position of the corresponding spinning nozzle, where $v=\frac{v_{\text {belt }}}{v_{\text {in }}} \geq 0$ defines the ratio between belt speed and spinning speed of the fiber, see [1]. The image of the fiber on the belt, denoted by $\left(\boldsymbol{\eta}_{t}\right)_{t \geq 0}$, is then obtained by $\boldsymbol{\eta}_{t}=\boldsymbol{\xi}_{t}-v t \mathbf{e}_{1}$. Unfortunately, there is no explicit computable stationary state available in case $v>0$. For a non-moving conveyor belt $(v=0)$ the equilibrium is given by (5).

\subsection{The 3D model}

In $[7,8,15]$ three-dimensional fiber lay-down models have been developed. Due to physical constraints, such as the inpenetrable conveyor belt or gravitation, the possibility of anisotropic fiber orientations in the resulting fiber web has to be taken into account. Therefore, it is necessary to amend the isotropic 3D models, i.e. (3) and (4) with $d=3$, $\boldsymbol{\theta}=(\alpha, \theta)$ and $\boldsymbol{\kappa}=\left(\kappa_{1}, \kappa_{2}\right)$ in order to enable this anisotropy. This has been realized via some weighting parameter $B \in[0,1]$. With the abbreviations

$$
\begin{aligned}
& p(\xi, \alpha, \theta):=\frac{1}{\sin \theta} \mathbf{n}_{1}(\alpha) \cdot \nabla V(\xi), \\
& q(\xi, \alpha, \theta):=\mathbf{n}_{2}(\alpha, \theta) \cdot \nabla V(\xi)
\end{aligned}
$$


one obtains the following (anisotropic) basic 3D model

$$
\begin{aligned}
& \mathrm{d} \boldsymbol{\xi}_{t}=\boldsymbol{\tau}\left(\alpha_{t}, \theta_{t}\right) \mathrm{d} t, \\
& \mathrm{~d} \alpha_{t}=-p\left(\xi_{t}, \alpha_{t}, \theta_{t}\right) \mathrm{d} t+\frac{1}{\sin \theta_{t}} A \mathrm{~d} W_{t}^{(1)}, \\
& \mathrm{d} \theta_{t}=-B q\left(\xi_{t}, \alpha_{t}, \theta_{t}\right) \mathrm{d} t+\frac{1}{2} A^{2} \cot \theta_{t} \mathrm{~d} t+A \sqrt{B} \mathrm{~d} W_{t}^{(2)}
\end{aligned}
$$

as well as the (anisotropic) smooth 3D model as

$$
\begin{aligned}
& \mathrm{d} \boldsymbol{\xi}_{t}=\boldsymbol{\tau}\left(\alpha_{t}, \theta_{t}\right) \mathrm{d} t, \\
& \mathrm{~d} \alpha_{t}=-p\left(\xi_{t}, \alpha_{t}, \theta_{t}\right) \mathrm{d} t+\frac{1}{\sin \theta_{t}} \kappa_{1, t} \mathrm{~d} t, \\
& \mathrm{~d} \theta_{t}=-B q\left(\xi_{t}, \alpha_{t}, \theta_{t}\right) \mathrm{d} t+B \kappa_{2, t} \mathrm{~d} t, \\
& \mathrm{~d} \kappa_{1, t}=p\left(\boldsymbol{\xi}_{t}, \alpha_{t}, \theta_{t}\right) \kappa_{2, t} \cos \theta_{t} \mathrm{~d} t-\kappa_{1, t} \kappa_{2, t} \cot \theta_{t} \mathrm{~d} t-\lambda \kappa_{1, t} \mathrm{~d} t+\mu \mathrm{d} W_{t}^{(1)}, \\
& \mathrm{d} \kappa_{2, t}=-p\left(\xi_{t}, \alpha_{t}, \theta_{t}\right) \kappa_{1, t} \cos \theta_{t} \mathrm{~d} t+\kappa_{1, t}^{2} \cot \theta_{t} \mathrm{~d} t-B \lambda \kappa_{2, t} \mathrm{~d} t+\mu \sqrt{B} \mathrm{~d} W_{t}^{(2)} .
\end{aligned}
$$

Here $\mathbf{W}=\left(W^{(1)}, W^{(2)}\right)$ denotes a standard two-dimensional Brownian motion. We note that for $B=0$ the respective 2D models (6), (7) are obtained. Basically, the weighting parameter allows for manipulation of the marginal $\theta$-distribution. The stationary solution of (9) is given by

$$
p_{s}\left(\xi, \theta, \kappa_{1}, \kappa_{2}\right)=C e^{-V_{B}(\xi)}(\sin \theta)^{\frac{1}{B}} e^{-\frac{\lambda}{\mu^{2}}\left(\kappa_{1}^{2}+\kappa_{2}^{2}\right)},
$$

compare also with Figure 3. Here $V_{B}:=(B+1) V$ denotes a rescaled potential. In the following we deal with this rescaled formulation, so we drop off the $B$ to facilitate the reading. The equilibrium state for the basic model (8) is obtained by integrating (10) over the curvatures $\left(\kappa_{1}, \kappa_{2}\right)$. Again the moving conveyor belt can be incorporated via

$$
\mathrm{d} \boldsymbol{\xi}_{t}=\boldsymbol{\tau}\left(\alpha_{t}, \theta_{t}\right) \mathrm{d} t+v \mathbf{e}_{1} \mathrm{~d} t
$$

Exemplary fiber trajectories computed with (9) for varying parameter $B$ with fixed parameters $\lambda=1, \mu=1, v=0$ and chosen potential $V(\xi)=\frac{1}{2}\|\xi\|^{2}$ are illustrated in Figures 4-6.

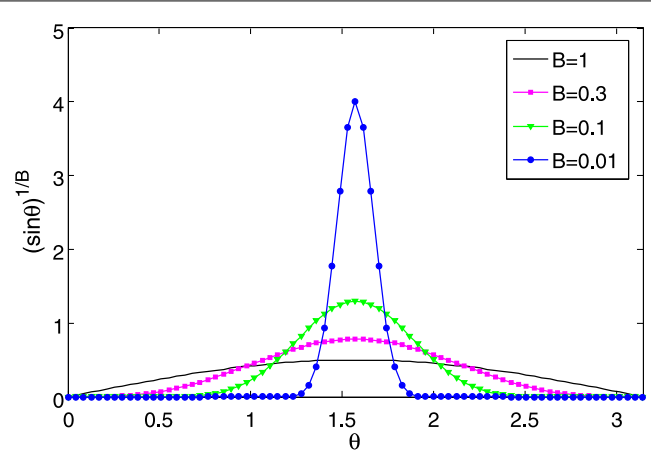

Figure 3 Behavior of the stationary $\theta$-density for decreasing $B$. 
Figure 4 Behavior of fiber trajectories in dependence on the weighting parameter. Here: $B=0.1$.

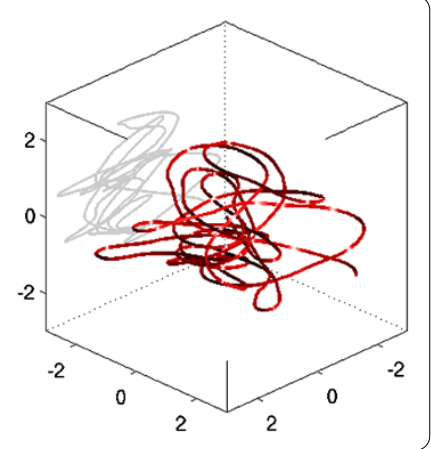

Figure 5 Behavior of fiber trajectories in dependence on the weighting parameter. Here: $B=0.01$

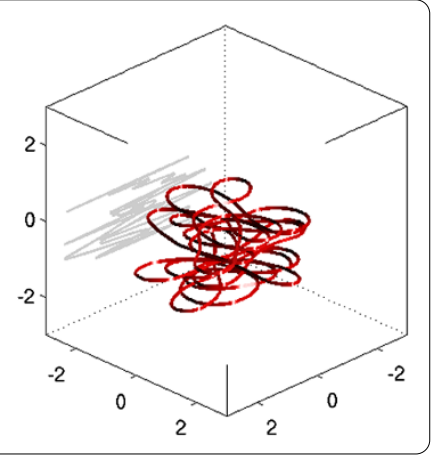

Figure 6 Behavior of fiber trajectories in dependence on the weighting parameter. Here: $B=0.001$

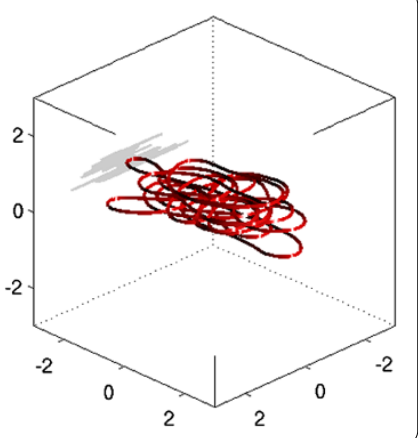

\section{Parameter estimation}

In this chapter, we specify our strategy to identify the parameters of the surrogate models. Here we focus on the calibration of the smooth 3D model (9) in the situation of a moving conveyor belt. More precisely, we follow the proposed approach from [8] that combine the heuristic identification method for the 2D models (compare [1]) with information about fiber orientations in the resulting non-woven gained from CT-scan measurements. The motivation for this approach is associated with the utilization of FIDYST as simulation tool of the physical fiber lay-down process. Its computations involve the motion of the fibers in turbulent air flows until they reach the conveyor belt where they are deposited. In this way FIDYST provides a two-dimensional texture that represents the fiber positions on the belt, see [17]. The entanglement of the fibers during their deposition is responsible for their typical forms, i.e. looping, buckling and coiling, see also [18, 19]. As already mentioned these characteristics are recovered by some parameters of the surrogate models. Apart from the supposedly known speed ratio $v$, we need estimations of the parameters $\mu, \lambda$ and 


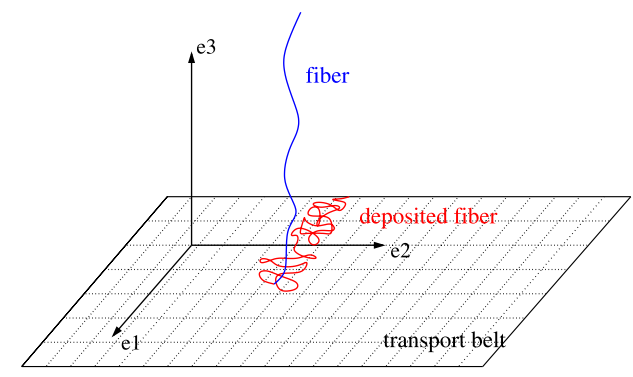

Figure 7 Sketch of fiber lay-down and fixing of the coordinate system.

$B$ as well as the shape of the potential $V$. We use a potential of the following form

$$
V(\xi)=\mathcal{V}\left(\xi_{1}, \xi_{2}\right)+\Phi\left(\xi_{3}\right), \quad \text { with } \mathcal{V}\left(\xi_{1}, \xi_{2}\right)=\frac{\xi_{1}^{2}}{2 \sigma_{1}^{2}}+\frac{\xi_{2}^{2}}{2 \sigma_{2}^{2}}
$$

where $\sigma_{1}, \sigma_{2}>0$ represents the standard deviations of the fiber throwing on the belt, compare Figure 7 for a specification of the coordinate system. Furthermore, $\Phi$ denotes a confining potential taking account of the location of the belt, such that $\xi_{3}$ is restricted to $\left[0, d_{f}\right]$, where $d_{f}$ denotes the thickness of the non-woven.

In summary, the parameters $\left(\sigma_{1}, \sigma_{2}, \mu, \lambda\right)$ are identified from a data sample of twodimensional fiber locations, denoted by $\boldsymbol{\eta}$ and provided by FIDYST, whereas the anisotropy parameter $B$ is obtained from $C T$-scans. For the following considerations, we suppose that the reference curve $\boldsymbol{\gamma}=-\nu \mathbf{e}_{1} t$ is known and that the deviation process $\boldsymbol{\xi}=\boldsymbol{\eta}-\boldsymbol{\gamma}$ is centered in the origin. At first, we dedicate ourselves to the afore-mentioned heuristic approach from [1] that enables the estimation of the 2D parameters. The advantage of this method lies in the use of the characteristic parameters that are actually observable in the process. Since the subsequently defined functional is closely related to the parameter space, the identification algorithm is very robust. An alternative method in the context of fiber laydown models using occupation times can be find in [20]. For more systematic approaches we refer to [21, 22]. Let $\mathcal{D}=\left(\mathbf{D}_{1}, \ldots, \mathbf{D}_{N}\right) \in\left(\mathbb{R}^{2} \times \mathbb{R} \times \mathbb{R}\right)^{N}$ with $\mathbf{D}_{i}=\left(\boldsymbol{\eta}_{t_{i}}, \alpha_{t_{i}}, \kappa_{t_{i}}\right), 1 \leq i \leq N$, be a set of data points obtained from a FIDYST simulation. Here an equidistant time grid with $\Delta t=t_{i+1}-t_{i}$ is used. The angles $\alpha_{t_{i}}$ and curvatures $\kappa_{t_{i}}$ are reconstructed from the fiber points $\boldsymbol{\eta}_{t_{i}}$ by finite difference approximations. We note that in [1] the emphasis is on the calibration of the model from [6], whereas we want to work with the smooth model (7) here. Therefore, with $\xi_{t}=\left(\xi_{t, 1}, \xi_{t, 2}\right)$, we consider a slightly different functional of characteristic properties than stated in [1]:

$$
\mathcal{F}(\mathcal{D})=\left(\mathcal{S}\left(\xi_{t, 1}\right), \mathcal{S}\left(\xi_{t, 2}\right), \mathcal{S}\left(\kappa_{t}\right), \mathcal{K}\left(\kappa_{t}\right)\right)
$$

where we define for a real valued stochastic process $\left(X_{t}\right)_{t \in\left\{t_{1}, \ldots, t_{N}\right\}}$,

$$
\begin{aligned}
& \mathcal{S}\left(X_{t}\right):=\sqrt{\frac{1}{N} \sum_{i=1}^{N}\left(X_{t_{i}}-\frac{1}{N} \sum_{j=1}^{N} X_{t_{j}}\right)^{2}}, \\
& \mathcal{K}\left(X_{t}\right):=\max _{k \in\{1, \ldots, \bar{k}\}} \sqrt{\frac{\sum_{i=1}^{N-k}\left(X_{t_{i+k}}-X_{t_{i}}\right)^{2}}{k(N-k) \triangle t}}, \quad \bar{k} \ll N,
\end{aligned}
$$


Table 1 Predetermined parameters by the pilot plant and size of data samples

\begin{tabular}{|c|c|c|c|c|c|c|}
\hline & \multicolumn{3}{|c|}{ Predetermined } & \multicolumn{2}{|l|}{ FIDYST } & \multirow{2}{*}{$\begin{array}{l}\text { IPD } \\
\text { \# points }\end{array}$} \\
\hline & $v_{\text {belt }}[\mathrm{m} / \mathrm{s}]$ & $v_{\text {spin }}[\mathrm{m} / \mathrm{s}]$ & $\boldsymbol{f}_{\text {diam }}[\mathrm{m}]$ & $\overline{\Delta t}[\mathrm{~m}]$ & \# points & \\
\hline Sample I & 0.633 & 79.400 & $12 \cdot 10^{-6}$ & 0.001 & $13 \times 19,850$ & $4,352,392$ \\
\hline Sample II & 4.167 & 79.400 & $12 \cdot 10^{-6}$ & 0.001 & $13 \times 17,850$ & 500,583 \\
\hline
\end{tabular}

which approximate the standard deviations of the processes and of their increments. In order to find the optimal calibration $\mathbf{P}=\left(\sigma_{1}, \sigma_{2}, \frac{\mu}{\sqrt{2 \lambda}}, \mu\right)$ of the surrogate model (7) with moving belt and potential $\mathcal{V}$, denoted by $\mathcal{D}_{\text {sur }}(\mathbf{P})$, we fix the minimization problem

$$
\mathbf{P}^{*}=\operatorname{argmin}_{\mathbf{P}}\left\|\mathcal{F}\left(\mathcal{D}_{\text {sur }}(\mathbf{P})\right)-\mathcal{F}\left(\mathcal{D}_{\text {fid }}\right)\right\|_{2}
$$

where $\mathcal{D}_{\text {fid }}$ indicates the data sample obtained from a FIDYST simulation. This can be solved by a relaxated quasi Newton method with unit Jacobian, i.e. $\mathbf{P}^{(n+1)}=\mathbf{P}^{(n)}+$ $\omega\left[\mathcal{F}\left(\mathcal{D}_{\text {sur }}\left(\mathbf{P}^{(n)}\right)\right)-\mathcal{F}\left(\mathcal{D}_{\text {fid }}\right)\right]$ and starting point $\mathbf{P}^{(0)}=\mathcal{F}\left(\mathcal{D}_{\text {fid }}\right)$, compare [1]. We note that $\mathcal{F}$ is a very good estimator for $\mathbf{P}$, if the fiber process is close to its stationary state (5) with $d=2$, i.e. for adequately large data sample and small speed ratio $v$, two conditions which are usually fulfilled in the production processes considered here. It is worth mentioning that this choice of $\mathcal{F}$ is completely detached from the white noise limit situation and can therefore be used for larger ranges of applications compared to [1].

We next describe how the data from CT-scan measurements of a non-woven can be handled to disclose the orientation of the fibers therein. This method of measurement is suitable for the examination, since the resolution of the CT-scan is around $1 \mu \mathrm{m}$, which is about an order of magnitude smaller than a typical fiber diameter, see Table 1 . The CTscan provides a real-valued three-dimensional matrix with so-called gray values. These raw image data are edited by image processing, which translates them into local fiber directions at each pixel with the help of an eigenvalue analysis of the Hessian matrix of the second partial derivatives of the gray values, for more details consult [23]. In other words, the image processing data (IPD) yield spherical polar angles that determine the orientation of the tangents at the fiber points. With these we generate the corresponding density distribution $\breve{p}^{(C T)}(\alpha, \theta)$ with $(\alpha, \theta) \in \breve{U}=\mathbb{R} / 2 \pi \mathbb{Z} \times\left(0, \frac{\pi}{2}\right]$. The restriction to the hemisphere is obligatory, since the data of the CT-scan do not reveal the temporal course of the (undistinguishable) fiber paths. Thus the tangents contain no more than an unsigned directional information. Therefore, the density $\breve{p}^{(C T)}(\alpha, \theta)$ has to be distinguished from the (unknown) angular density distribution obtained by our model (9) with moving conveyor belt, in the following denoted by $p^{(M)}(\alpha, \theta)$ with $(\alpha, \theta) \in U=\mathbb{R} / 2 \pi \mathbb{Z} \times(0, \pi)$. The latter can be convert to the hemisphere via

$$
\breve{p}^{(M)}(\alpha, \theta)=\frac{1}{2}\left(p^{(M)}(\alpha, \theta)+p^{(M)}(\alpha+\pi, \pi-\theta)\right), \quad(\alpha, \theta) \in \breve{U}
$$

which allows us to compare the densities.

Assuming that the fiber lay-down process is close to equilibrium, or in other words, if $v$ is sufficiently small, we can identify the parameter $B$ without great effort. In that case, we demand the equality of the standard deviation $\sigma_{B}$ of the $\theta$-marginal of the distribution function obtained from the CT-scan, denoted by $\breve{p}_{\theta}^{(C T)}$, and the explicitly computed standard deviation $\bar{\sigma}_{B}$ of the $\theta$-marginal of the stationary distribution (10) of our model (with $v=0$ ). The latter reads $\bar{\sigma}_{B}=\left(\int_{0}^{\pi} C_{B}(\sin \theta)^{\frac{1}{B}}\left(\theta-\frac{\pi}{2}\right)^{2} \mathrm{~d} \theta\right)^{\frac{1}{2}}$ with normalization constant 
$C_{B}^{-1}=\int_{0}^{\pi}(\sin \theta)^{\frac{1}{B}} \mathrm{~d} \theta$. Besides, the influence of the belt speed on the size of $B$ is expected to be small in contrast to the other parameters, since it involves the deviation from the planar structure. In the case of very large $v$, the estimation of $B$ can be straightforwardly embedded in the above heuristic identification approach with a little more effort. For our purposes, however, the described identification strategy will turn out to be adequate anyway.

\section{Application}

In the following we want to demonstrate the adaptation of the surrogate models on the basis of a industrial test case describing a real non-woven production process. From a pilot plant of the company Oerlikon Neumag process data have been available that have been used to initialize FIDYST simulations of the full process. Plant specific parameters, such as machine geometry and prevailing air flows have been included in these computations. In addition, an associated non-woven product has been produced by the pilot plant with the same configuration data. Pieces of this non-woven have been cut and have been analyzed in CT-scans. The statistics of the fiber orientation in the non-woven given as image processing data (IPD) complement the FIDYST informations as described in the previous chapter.

We discuss two different data samples, denoted by sample $I$ and sample $I I$. Associated characteristic values and data sizes are summarized in Table 1. The resolution of FIDYST is something to be viewed critically. A more accurate resolution increases the effort and leads to unreasonable computational costs, compare Chapter 1. At least the surrogate models use the same grid sizes and they can be viewed as optimal discrete substitutes for FIDYST, see also below. The different numbers of IPD points indicate a different usable sample size of the pieces of nonwoven. A larger number of points leads to smoother $\alpha$-marginals $\breve{p}_{\alpha}^{(C T)}(\alpha, \theta)$, compare Figures 8,9 , otherwise, it is not relevant. The essential difference be-

Figure 8 Simulated fibers (red) with one highlighted filament (blue). Here: FIDYST, sample I. highlighted filament (blue). Here: surrogate model, sample I.
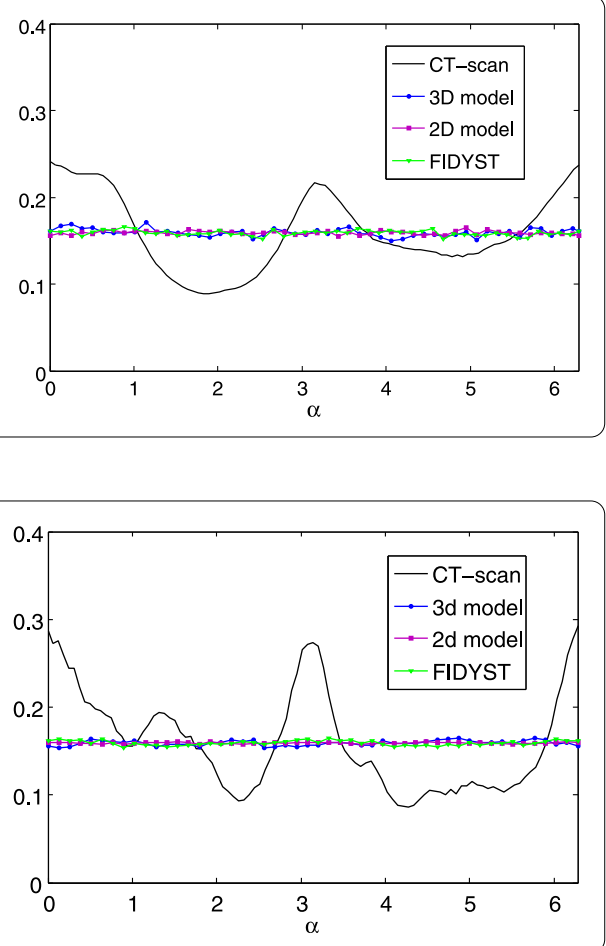
Table 2 Identified parameters to be used in the surrogate model

\begin{tabular}{lllllll}
\hline & $\boldsymbol{\sigma}_{\mathbf{1}}[\mathbf{m}]$ & $\boldsymbol{\sigma}_{\mathbf{2}}[\mathbf{m}]$ & $\boldsymbol{\mu}\left[\mathbf{m}^{-\mathbf{3} / \mathbf{2}}\right]$ & $\boldsymbol{\lambda}\left[\mathbf{m}^{-\mathbf{1}}\right]$ & $\boldsymbol{B}$ & $\boldsymbol{v}$ \\
\hline Sample I & 0.0050 & 0.0049 & 49,096 & 1,039 & 0.398 & 0.0080 \\
Sample /I & 0.0055 & 0.0045 & 49,885 & 997 & 0.375 & 0.0525 \\
\hline
\end{tabular}

Figure 10 Simulated fibers (red) with one highlighted filament (blue). Here: FIDYST, sample II.

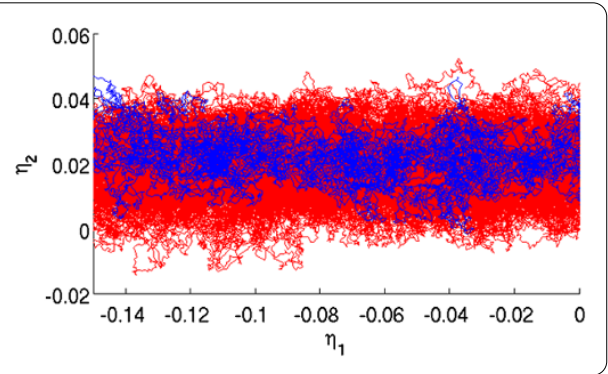

Figure 11 Simulated fibers (red) with one highlighted filament (blue). Here: surrogate model, sample II.

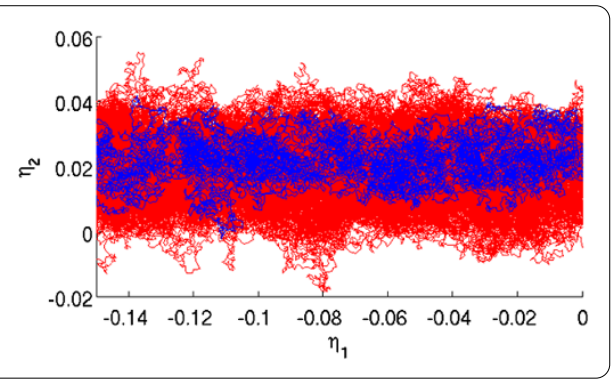

Figure 12 Marginal angular distribution $\breve{p}_{\theta}^{(C T)}(\alpha$, $\boldsymbol{\theta}), \breve{\boldsymbol{p}}_{\boldsymbol{\theta}}^{(\boldsymbol{M})}(\boldsymbol{\alpha}, \boldsymbol{\theta})$. Here: sample I.

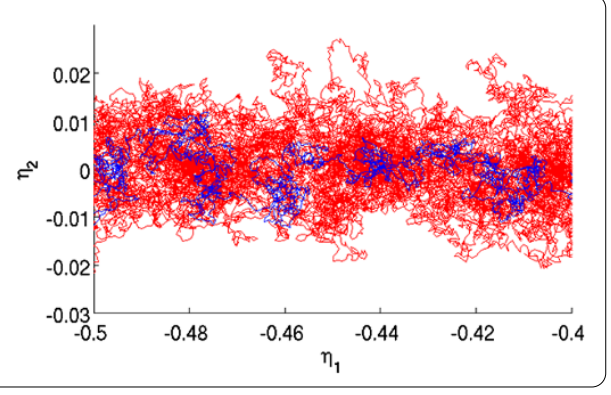

tween the two samples is the belt speed. Nevertheless, the speed ratio $v$ is sufficiently small in both cases, which can be ascertained by the comparison between the standard deviation $\bar{\sigma}_{B}$ and the one of our model with given $v$ using Monte-Carlo simulations. Thus the calibration of the surrogate model (9) is carried out as described above. The estimated parameters are summarized in Table 2. In Figures 10-13 we illustrate a comparison of fibers computed by FIDYST and the calibrated 2D model (7) with moving transport belt. Qualitatively the same fiber lay-down structures are observed. The parameter $B$ is identified from the CT-scans data as outlined above. This leads to $\theta$-marginal distributions as in Figures 14, 15.

Furthermore, in Figures 8, 9 we compare the $\alpha$-marginal $\breve{p}_{\alpha}^{(C T)}(\alpha, \theta)$ of the CT-scan data with the corresponding distributions obtained from FIDYST simulations $\breve{p}_{\alpha}^{\left(M_{F}\right)}(\alpha, \theta)$ and from the surrogate models $\breve{p}_{\alpha}^{\left(M_{2 d}\right)}(\alpha, \theta)$ and $\breve{p}_{\alpha}^{\left(M_{3 d}\right)}(\alpha, \theta)$, which can be computed with the help of (11), (where $\theta=\pi / 2$ is fixed for $p^{\left(M_{F}\right)}$ and $p^{\left(M_{2 d}\right)}$ ). It seems conspicious, that the large amplitude in the CT-scans is not reached by the models. On the contrary, both FIDYST 
Figure 13 Marginal angular distribution $\breve{p}_{\theta}^{(C T)}(\alpha$, $\boldsymbol{\theta}), \breve{\boldsymbol{p}}_{\boldsymbol{\theta}}^{(\boldsymbol{M})}(\boldsymbol{\alpha}, \boldsymbol{\theta})$. Here: sample II.

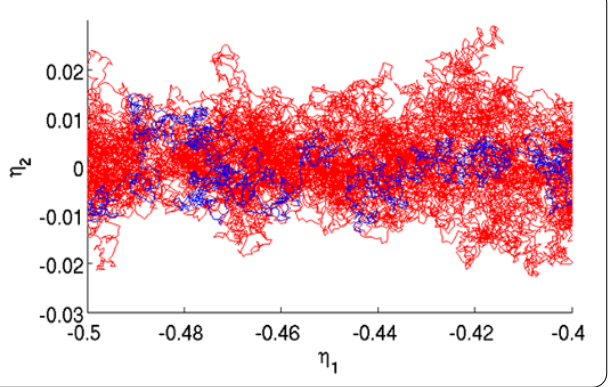

Figure 14 Marginal angular distribution $\breve{p}_{\alpha}^{(C T)}(\alpha, \theta)$, $\breve{p}_{\boldsymbol{\alpha}}^{(M)}(\boldsymbol{\alpha}, \boldsymbol{\theta})$. Here: sample I.

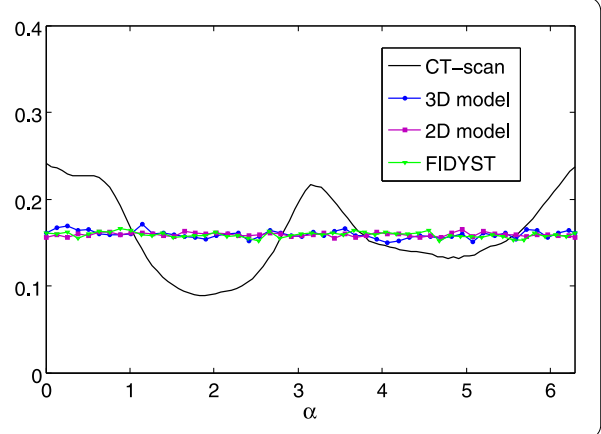

Figure 15 Marginal angular distribution $\breve{p}_{\alpha}^{(C T)}(\alpha, \theta)$, $\breve{p}_{\alpha}^{(M)}(\boldsymbol{\alpha}, \boldsymbol{\theta})$. Here: sample II.

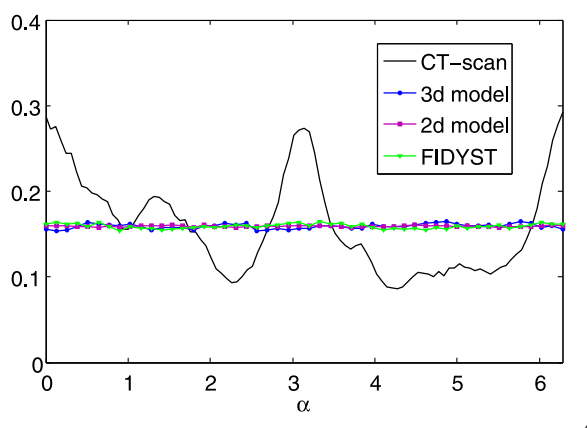

and the surrogate models (7) and (9) show an almost uniform distribution, which is not surprising because of the very small speed ratio. Indeed, the surrogate models show the same quantitative behavior as the FIDYST simulation.

This discrepancy between the CT-scan measurements and the models with respect to the $\alpha$-distribution might be explained by the fact that the CT-scan has analyzed pieces of non-woven that had already passed through several process steps of reworking and reinforcement which had influenced the angular distribution. On the contrary, both, FIDYST and the surrogate fiber lay-down models have described the texture before postprocessing. To investigate this issue more closely, it would be of great interest to have CT-scans of the deposited fibers without effects due to post-processing.

Finally, we use the calibrated 3D model to simulate virtual fiber webs by superposing hundred fibers. The distance of neighboring spinning positions $a \approx 0.0015 \mathrm{~m}$ can be determined from FIDYST simulations, whereas the nonwoven thickness $d_{f}$ has to be appropriately chosen in the potential $V$. Unfortunately, it is technically impossible to get at this parameter. We can only estimate in terms of the fiber diameter $f_{\text {diam }}$. Exemplary simulations for the two samples with a presumed non-woven thickness $d_{f}=200 f_{\text {diam }}$ are shown 


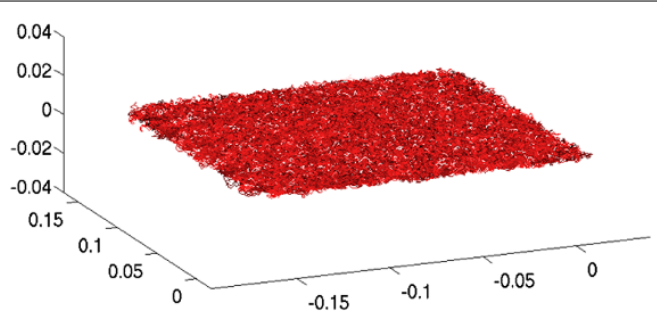

Figure 16 Simulation of a fiber web corresponding to sample $I$. Here: side view.

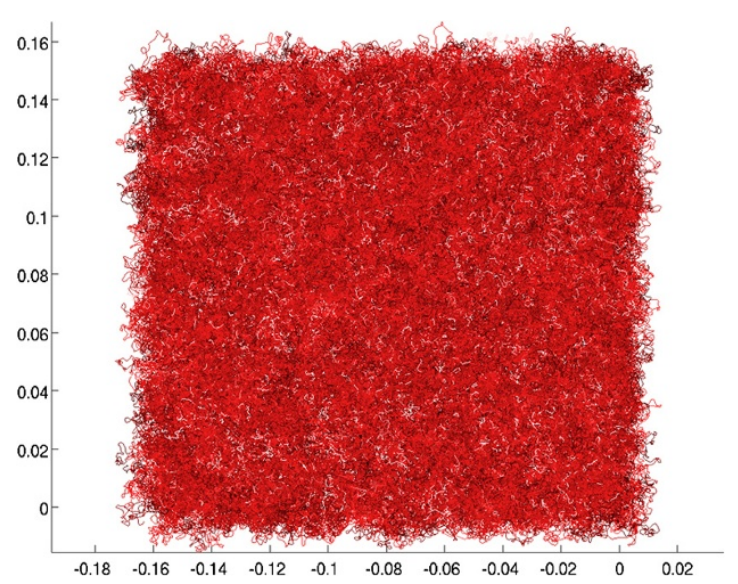

Figure 17 Simulation of a fiber web corresponding to sample $I$. Here: top view.

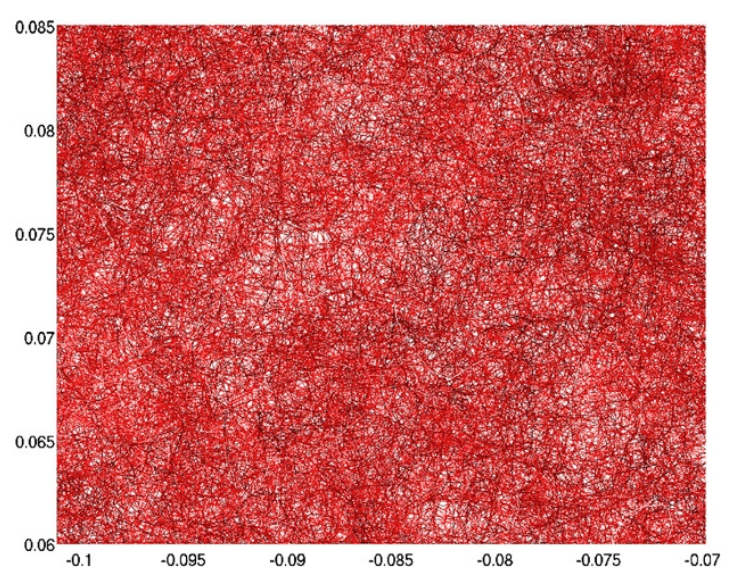

Figure 18 Simulation of a fiber web corresponding to sample $\mathbf{l}$. Here: top view (zoomed in).

in Figures 16-21. To compare the quality of the resulting fiber webs, we investigate the homogeneity more closely. As is common in the practical application, we look at the basis weight distribution. For this purpose, a 2D grid is placed over a fixed area of the simulated fiber web (projected to 2D) and the basis weight of each cell is numerically determined. This weight $M$ of a given cell is proportional to the time the fiber process is present in that cell. In Figures 22-27 the relative deviations $\frac{|E[M]-M|}{E[M]}$ between the (expected) averaged and the actual basis weight in the cells are illustrated for different grid sizes. The standard de- 


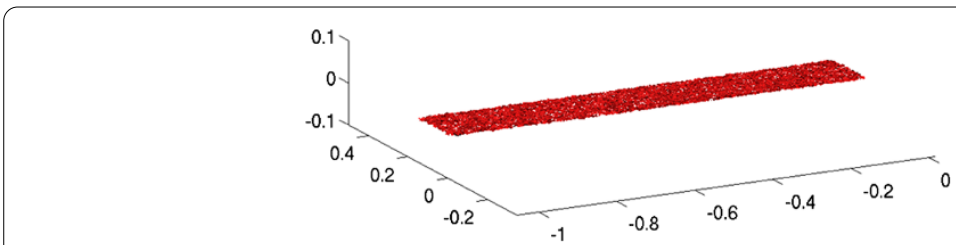

Figure 19 Simulation of a fiber web corresponding to sample II. Here: side view.

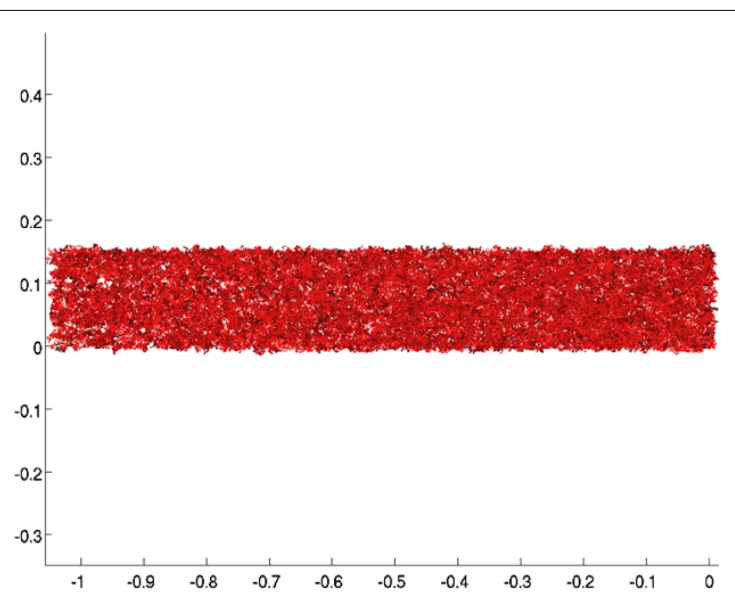

Figure 20 Simulation of a fiber web corresponding to sample II. Here: top view.

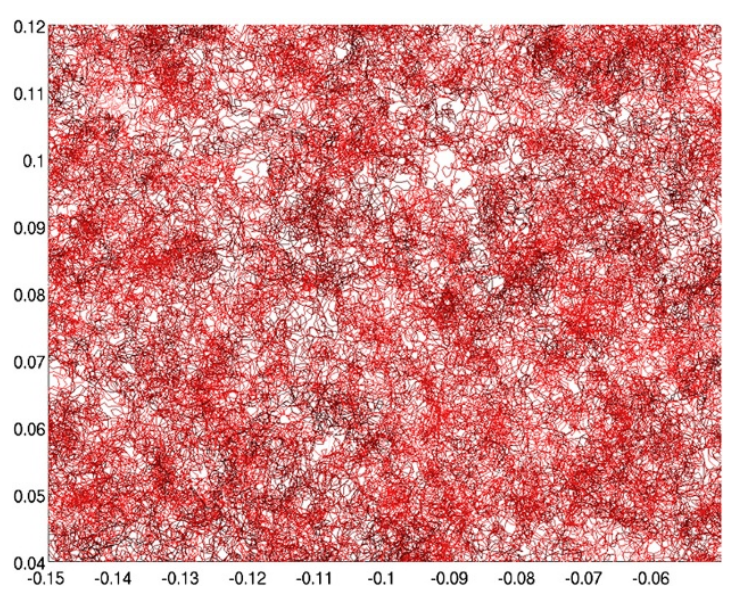

Figure 21 Simulation of a fiber web corresponding to sample II. Here: top view (zoomed in).

viations of these values give the corresponding coefficients of variation ( $C V$-values) which are summarized in Table 3. As specimen we choose a square of size $100 \mathrm{~cm}^{2}$ for each sample. Due to the respective smaller $C V$-values we can state that the simulated fiber web associated to sample $I$ is more homogeneous than the one given by sample $I I$ and hence better quality characteristics of nonwovens are expected for the machine configuration of sample $I$, i.e. for a slower conveyor belt. Due to the different belt speed, however, the basis weight with respect to sample $I I$ is six times smaller compared to sample $I$. 


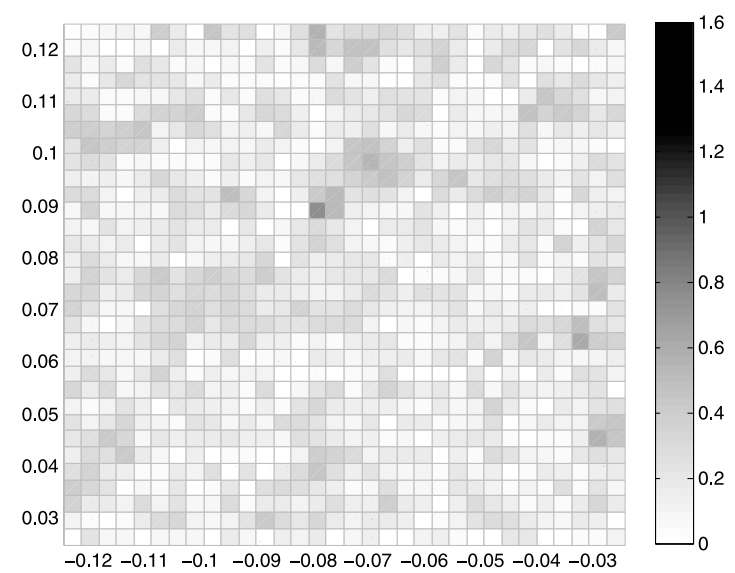

Figure 22 Relative deviation of basis weight per grid cell. Here: sample I, $32 \times 32$-grid.

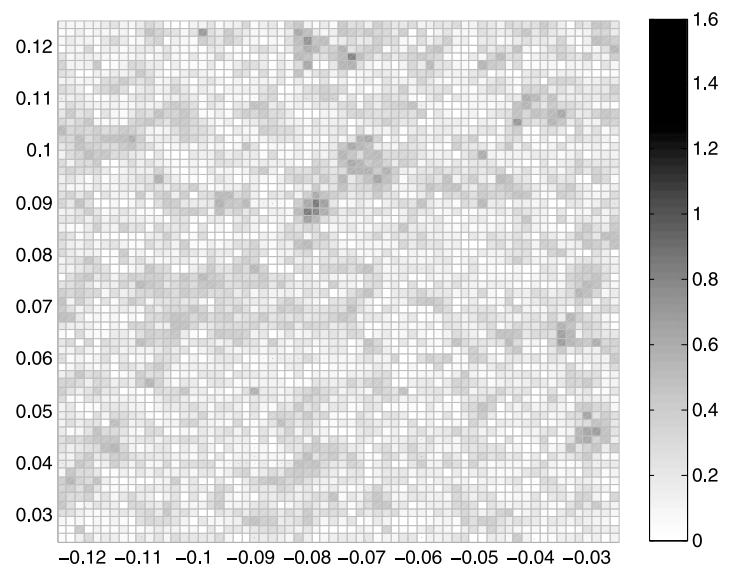

Figure 23 Relative deviation of basis weight per grid cell. Here: sample I, $64 \times 64$-grid.

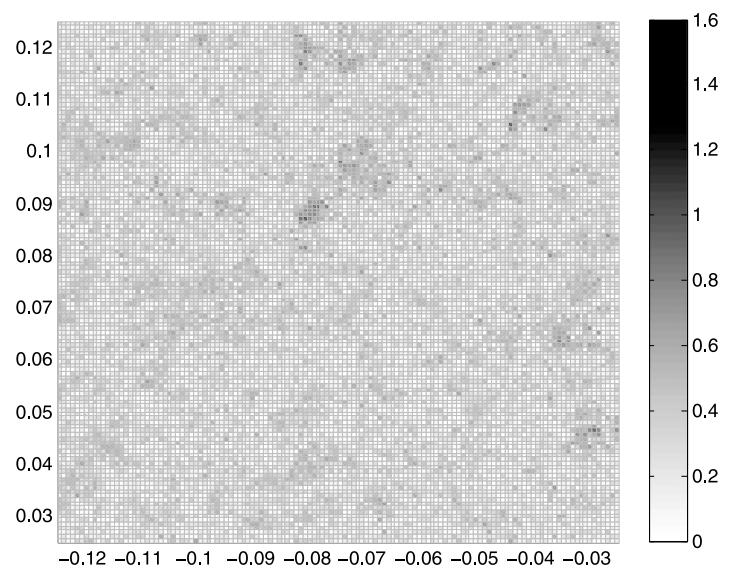

Figure 24 Relative deviation of basis weight per grid cell. Here: sample /, 128 × 128-grid. 


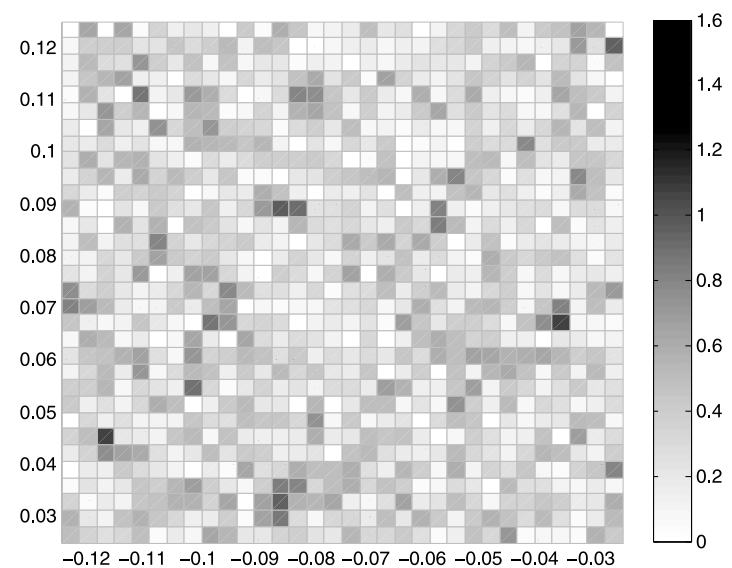

Figure 25 Relative deviation of basis weight per grid cell. Here: sample II, $32 \times 32$-grid.

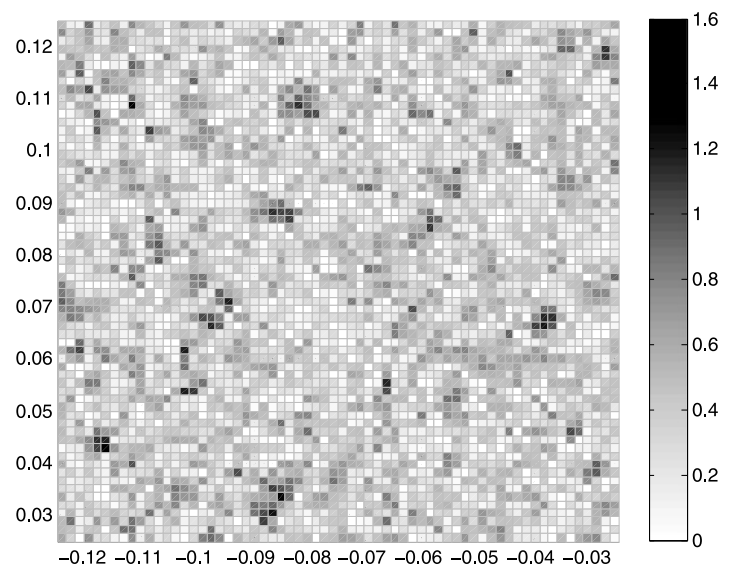

Figure 26 Relative deviation of basis weight per grid cell. Here: sample II, $64 \times 64$-grid.

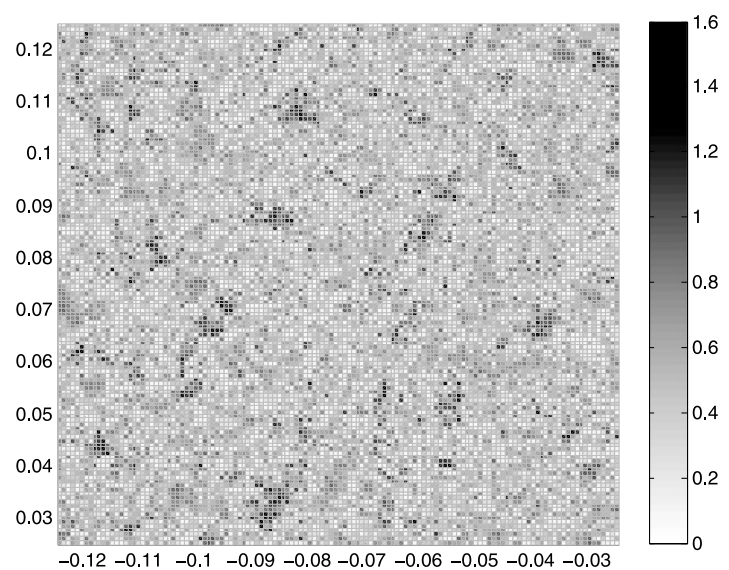

Figure 27 Relative deviation of basis weight per grid cell. Here: sample II, $128 \times 128$-grid. 
Table 3 Basis weight distribution in terms of CV-values (in \%) of the simulated fiber webs

\begin{tabular}{llll}
\hline & Grid & & \\
\cline { 2 - 4 } & $\mathbf{3 2 \times 3 2}$ & $\mathbf{6 4} \times \mathbf{6 4}$ & $\mathbf{1 2 8} \times \mathbf{1 2 8}$ \\
\hline Sample I & 16.4 & 18.9 & 22.6 \\
Sample II & 31.8 & 38.9 & 49.1 \\
\hline
\end{tabular}

\section{Conclusion and outlook}

We presented the application of a 3D surrogate fiber lay-down model to an industrial problem. The parameters are identified on the basis of experimental data. The calibrated model enables the efficient simulation of a whole virtual fiber web. Further modifications of the model will include, for example, the impenetrability of the fibers. This is examined in further studies. To predict material properties like permeability of the textile, the geometric model presented here has to be used as a basic model for a complex flow simulation to determine macroscopic properties of the textile.

\section{Competing interests}

The authors declare that they have no competing interests.

\section{Authors' contributions}

All authors contributed equally to the writing of this paper. All authors read and approved the final manuscript.

\section{Author details}

${ }^{1}$ Technische Universität Kaiserslautern, Gottlieb-Daimler Str., Kaiserslautern, 67663, Germany. ${ }^{2}$ Fraunhofer-Institut für Techno- und Wirtschaftsmathematik, Fraunhofer Platz 1, Kaiserslautern, 67663, Germany.

\section{Acknowledgements}

This work has been supported by Bundesministerium für Bildung und Forschung (BMBF), Verbundprojekt ProFil, 03 MS606. Moreover, we thank the colleagues from the departments Transport Processes and Image Processing at Fraunhofer ITWM.

Endnote

a FIDYST: Fiber Dynamics Simulation Tool developed at Fraunhofer ITWM, Kaiserslautern.

Received: 7 May 2012 Accepted: 19 March 2014 Published: 03 Jun 2014

References

1. Klar A, Marheineke N, Wegener R: Hierarchy of mathematical models for production processes of technical textiles. ZAngew Math Mech 2009, 89(12):941-961.

2. Marheineke N, Wegener R: Fiber dynamics in turbulent flows: general modeling framework. SIAM J App/ Math 2006, 66(5):1703-1726.

3. Marheineke N, Wegener R: Modeling and application of a stochastic drag for fibers in turbulent flows. Int J Multiph Flow 2011, 37:136-148.

4. Götz T, Klar A, Marheineke N, Wegener R: A stochastic model and associated Fokker-Planck equation for the fiber lay-down process in nonwoven production processes. SIAM J App/ Math 2007, 67(6):1704-1717.

5. Bonilla LL, Götz T, Klar A, Marheineke N, Wegener R: Hydrodynamic limit of a Fokker-Planck equation describing fiber lay-down processes. SIAM J Appl Math 2007/08, 68(3):648-665.

6. Herty M, Klar A, Motsch S, Olawsky F: A smooth model for fiber lay-down processes and its diffusion approximations. Kinet Relat Models 2009, 2(3):489-502.

7. Klar A, Maringer J, Wegener R: A smooth 3D model for fiber lay-down in nonwoven production processes. Kinet Relat Models 2012, 5:97-112.

8. Klar A, Maringer J, Wegener R: A 3D model for fiber lay-down in nonwoven production processes. Math Models Methods App/ Sci 2012, 22(9):1250020.

9. Grothaus M, Klar A: Ergodicity and rate of convergence for a nonsectorial fiber lay-down process. SIAM J Math Anal 2008, 40(3):968-983.

10. Dolbeault J, Klar A, Mouhot C, Schmeiser C: Exponential rate of convergence to equilibrium for a model describing fiber lay-down processes. Appl Math Res Express 2012. doi:10.1093/amrx/abs015.

11. Kolb M, Savov M, Wübker A: (Non-)ergodicity of a degenerate diffusion modeling the fiber lay down process. SIAM J Math Anal 2013, 45:1-13.

12. Kolb M, Savov M, Wübker A: Geometric ergodicity of a hypoelliptic diffusion modelling the melt-spinning process of nonwoven materials; 2012 [arXiv:1112.6159]

13. Grothaus M, Klar A, Maringer J, Stilgenbauer P: Geometry, mixing properties and hypocoercivity of a degenerate diffusion arising in technical textile industry; 2012 [arXiv:1203.4502]

14. Grothaus M, Stilgenbauer P: Hypocoercivity for Kolmogorov backward evolution equations and applications; 2012 [arXiv:1207.5447] 
15. Grothaus M, Stilgenbauer P: Geometric Langevin equations on submanifolds and applications to the stochastic melt-spinning process of nonwovens and biology. Stoch Dyn 2013, 13(4):1350001.

16. Hsu EP: Stochastic Analysis on Manifolds. Providence: Am. Math. Soc.; 2002. [Graduate Studies in Mathematics]

17. Hietel D, Wegener R: Simulation of spinning and lay-down processes. Tech Text 2005, 3:145-148.

18. Hearle J, Sultan M, Govender S: The form taken by threads laid on a moving belt. J Text Inst 1976, 67:373-386.

19. Mahadevan L, Keller J: Coiling of flexible ropes. Proc R Soc Lond A 1996, 452(1950):1679-1694.

20. Bock W, Götz T, Liyanage P: Parameter estimation of fiber lay-down in nonwoven production - an occupation time approach; 2011 [arXiv:1112.3550]

21. Kutoyants YA: Statistical Inference for Ergodic Diffusion Processes. London: Springer; 2004. [Springer Series in Statistics]

22. Gobet E, Hoffmann M, Reiß M: Nonparametric estimation of scalar diffusions based on low frequency data. Ann Stat 2004, 32(5):2223-2253.

23. Redenbach C, Rack A, Schladitz K, Wirjadi O, Godehardt M: Beyond imaging: on the quantitative analysis of tomographic volume data. Int J Mater Res 2012, 2:217-227.

10.1186/2190-5983-4-4

Cite this article as: Grothaus et al.: Application of a three-dimensional fiber lay-down model to non-woven

production processes. Journal of Mathematics in Industry 2014, 4:4

\section{Submit your manuscript to a SpringerOpen ${ }^{\ominus}$ journal and benefit from:}

- Convenient online submission

- Rigorous peer review

- Immediate publication on acceptance

Open access: articles freely available online

High visibility within the field

- Retaining the copyright to your article 